\title{
EFEITO DA HIDRÓLISE ÁCIDA DOS TANINOS DE Eucalyptus grandis W. Hill ex Maiden NAS PROPRIEDADES DOS ADESIVOS TÂNICOS ${ }^{1}$
}

\author{
Angélica de Cássia Oliveira Carneiro², Benedito Rocha Vital ${ }^{2}$, Pedro Gustavo Ulisses Frederico ${ }^{3}$, \\ Rosimeire Cavalcante dos Santos ${ }^{4}$ e Wagner Patrício de Sousa Júnior ${ }^{5}$

\begin{abstract}
RESUMO - Este trabalho teve como objetivo avaliar o efeito da hidrólise ácida sobre as propriedades e resistência ao cisalhamento dos adesivos de taninos, extraídos a partir da casca de Eucalyptus grandis W. Hill ex Maiden. Os adesivos foram sintetizados com taninos hidrolisados, empregando-se quatro valores de $\mathrm{pH}$, três tempos de reação e $10 \%$ de formaldeído em relação à massa seca de taninos. Foram produzidas 96 juntas coladas, constituídas de duas lâminas de madeira de Eucalyptus grandis. As resistências ao cisalhamento e falha na madeira foram determinadas de acordo com a norma ASTM D 2339-93. Concluiu-se que a hidrólise ácida dos taninos reduziu a viscosidade dos adesivos e aumentou a resistência ao cisalhamento na linha de cola. De modo geral, as juntas coladas apresentaram baixo percentual de falha na madeira.
\end{abstract}

Palavras-chave: Cascas, taninos e adesivos.

\section{EFFECT OF THE ACID HYDROLYSES OF Eucalyptus grandis W. Hill ex Maiden TANNINS IN THE PROPERTIES OF THE TANNIC ADHESIVES}

\begin{abstract}
The objective of this research was to evaluate the effect of the acid hydrolysis of Eucalyptus grandis W. Hill ex Maiden bark tannins on the properties of tannic adhesives. Adhesives were synthesized with tannins hydrolyzed at four $\mathrm{pH}$ values, three reaction times and $10 \%$ of formaldehyde based on the tannin dry weight. Ninety-six glued joints were prepared with Eucalyptus grandis thin boards. Shear resistance and wood failure percentage were determined according to the ASTM D 2339-93 standards. It was concluded that tannin hydrolysis decreased adhesive viscosity and increased the glue line shear resistance. However, it was observed a low percentage of wood failure.
\end{abstract}

Keywords: Bark, tannins and adhesives.

\section{INTRODUÇÃ̃O}

De acordo com Simão (1992), o eucalipto, em virtude de seu rápido crescimento nas mais variadas condições de solo e clima e das múltiplas possibilidades de utilização de sua madeira, tem-se tornado a essência florestal mais usada no Brasil, principalmente pelas indústrias de carvoejamento, madeireiras e de celulose e papel. As atividades dessas empresas levam à produção de grande quantidade de resíduos, destacando-se o material proveniente do descascamento das toras das árvores (cascas), o que gera problemas para a empresa, como por a ocupação de áreas, os aspectos estéticos e, sobretudo, o alto risco de incêndio.

As cascas de Eucalyptus sp. são, assim, um subproduto ou, mesmo, um resíduo para a maioria das indústrias madeireiras, trazendo problemas de descarte e de ordens ambiental e econômica, uma vez que milhares de toneladas de casca são produzidos anualmente pelas indústrias que consomem madeira de eucalipto.

\footnotetext{
${ }^{1}$ Recebido em 07.10.2007 e aceito para publicação em 29.05.2009.

${ }^{2}$ Departamento de Engenharia Florestal da Universidade Federal de Viçosa (UFV). E-mail: <cassiacarneiro@ufv.br>, <bvital@ufv.br>. ${ }^{3}$ Programa de Pós-Graduação em Ciência Florestal da UFV.

${ }^{4}$ Programa de Pós-Graduação em Ciência Florestal da Universidade Federal de Lavras (UFLA). E-mail: <meire_caico@ yahoo.com.br>.

${ }^{5}$ Tubominas Indústria e Comércio Ltda. E-mail: <wagner@ tubominas.com.br>.
} 
Uma alternativa de uso do volume de casca excedente nas fábricas seria a extração de taninos para produção de adesivos para madeira, e, após a sua extração, elas poderiam ser queimadas nas caldeiras ou, ainda, ser utilizadas em compostagem. Essas cascas também poderiam ser utilizadas para produção de chapas de composição.

Os adesivos à base de tanino-formaldeído apresentam algumas limitações. Os extratos de taninos contêm, além de substâncias fenólicas ativas, substâncias como traços de aceno e aminoácidos e, principalmente, açúcares e gomas de alto peso molecular, o que contribui para a diminuição da qualidade da colagem. Em geral, os adesivos à base de taninos de eucalipto apresentam alta viscosidade e vida útil de trabalho curta, acarretando problemas de aplicação industrial, entretanto tais dificuldades podem ser contornadas.

Existem alternativas para melhorar as propriedades dos adesivos à base de taninos, mas nem sempre elas são aplicadas com sucesso, em razão, principalmente, da diferença do conteúdo fenólico entre as espécies. Entre estas, destacam-se a sulfitação dos taninos e a hidrólise ácida ou alcalina.

A hidrólise de taninos para a produção de adesivos tânicos vem sendo estudada por vários pesquisadores (MORI, 2000; SOWUNMI et al., 2000; BISANDA et al., 2003; CARNEIRO et al., 2004), porém a redução da viscosidade, mantendo-se a alta resistência da junta colada, ainda não foi alcançada.

A hidrólise ácida, atualmente, é a técnica mais utilizada na síntese dos adesivos tânicos, pois tem sido aplicada para quebrar as ligações interflavonoides e, também, abrir o anel heterocíclico, ocorrendo, assim, a formação de um carbon-cátion, com capacidade de reagir com outros nucleofílicos presentes (SOWUNMI et al., 2000) (Figura 1).
A hidrólise reduz o tamanho molecular do componente flavonoide, consequentemente as moléculas ficam com maior mobilidade e os extratos tânicos ficam, também, livres de gomas, que foram hidrolisadas, reduzindo a viscosidade dos adesivos, permitindo, assim, que a adesão química se torne mais eficiente entre as moléculas polifenólicas e o agente ligante, formaldeído ou paraformaldeído (SOWUNMI et al., 1996, 2000).

Diante disso, o objetivo principal deste estudo foi avaliar o efeito do tempo de reação e pH de hidrólise sobre as propriedades e a resistência ao cisalhamento dos adesivos de taninos, extraídos a partir da casca de Eucalyptus grandis.

\section{MATERIAL E MÉTODOS}

\subsection{Extração dos taninos}

Os taninos empregados nesta pesquisa foram extraídos da casca de Eucalyptus grandis, provenientes de plantios da Universidade Federal de Viçosa em Viçosa, MG, com idade de 20 anos. As cascas foram moídas em moinho de martelo e, em seguida, peneiradas, recolhendo-se a fração que passou na peneira de 40 mesh e foi retida na de $60 \mathrm{mesh}$. Depois de peneiradas, foram acondicionadas em sacos plásticos, com teor de umidade médio de $15 \%$.

Na extração dos taninos, utilizou-se a metodologia empregada por Carneiro (2002), por ter apresentado maior rendimento em taninos. A extração foi feita em autoclave à temperatura de $70^{\circ} \mathrm{C}$, por um período de $3 \mathrm{~h}$, adicionandose $4,5 \%$ de sulfito de sódio à água de extração. Utilizouse uma relação licor/casca de 20:1. Os extratos tânicos foram distribuídos em bandejas de alumínio e levados à estufa a uma temperatura de $70^{\circ} \mathrm{C}$ até a secagem total. Os taninos foram moídos até a granulometria de 100 mesh, para posterior produção dos adesivos.<smiles>Oc1cc(O)c2c(c1)OC(c1cc(O)c(O)c(O)c1)C(O)C2</smiles><smiles>Oc1cc(O)c(CCCc2cc(O)c(O)c(O)c2)c(O)c1</smiles>

Fonte: SOWUNMI et al., 2000. Source: SOWUNMI et al., 2000.

Figura 1 - Mecanismo de hidrólise ácida dos taninos. Figure 1 - Tannic acid hydrolysis mechanism. 


\subsection{Hidrólise dos taninos}

Os taninos em pó foram solubilizados em água ( solução a 50\% de sólidos) e hidrolisados com ácido acético concentrado. O controle da extensão da hidrólise foi feito, ajustando-se o pH dos taninos e o tempo de reação. Empregaram-se tempos de 30, 60 e 90 min e os seguintes valores de $\mathrm{pH}: 3,4,5$ e 6 ( $\mathrm{pH}$ dos taninos não hidrolisados).

\subsection{Produção dos adesivos}

Para a produção de adesivos, foram utilizadas as soluções aquosas contendo os taninos hidrolisados e, também, os taninos brutos, ou seja, sem modificação química. Para tanto, os taninos foram misturados em solução com formaldeído, agitando-o com um bastão de vidro por $1 \mathrm{~min}$. Em todos os tratamentos, utilizaram-se $10 \%$ de formaldeído em relação à massa seca de sólidos.

\subsection{Propriedades dos adesivos}

O teor de sólidos dos adesivos foi calculado de acordo com Moslemi (1974), evaporando-se a água das amostras, contendo $3 \mathrm{~g}$ de adesivo, que foram levados à estufa até atingir peso constante.

O tempo de gelatinização foi obtido com amostras de $1 \mathrm{~g}$ do adesivo, colocadas em tubos de ensaio de 15 cm de alturae 2 cm de diâmetro, em cujo interior foi mergulhado um bastão de vidro. O conjunto tubo-bastão foi aquecido até $170^{\circ} \mathrm{C}$, no aparelho gel-timer, cronometrando-se o tempo gasto para a polimerização do adesivo.

A viscosidade foi obtida por um viscosímetro Brookfield-LV (splinder 3), empregando-se uma velocidade de rotação de $12 \mathrm{rpm}$, em amostras de aproximadamente $300 \mathrm{~mL}$, em três repetições, na temperatura de $25^{\circ} \mathrm{C}$.

\subsection{Colagem das lâminas}

Para determinar a resistência da linha de cola ao cisalhamento e a porcentagem de falha na madeira, foram produzidas 96 juntas, constituídas de duas lâminas de Eucalyptus grandis. A espessura média das lâminas foi de $0,60 \mathrm{~cm}$ e a umidade média, em torno de $8,0 \%$. As juntas foram produzidas com dimensões finais de $38 \times 10 \times 0,6 \mathrm{~cm}$, aplicando-se $250 \mathrm{~g} / \mathrm{m}^{2}$ de adesivo em face dupla. As juntas foram prensadas a $12 \mathrm{kgf} / \mathrm{cm}^{2}$ e temperatura de $170{ }^{\circ} \mathrm{C}$, durante $8 \mathrm{~min}$. Depois de prensadas, as juntas coladas foram condicionadas à temperatura ambiente até que alcançassem a umidade de equilíbrio $( \pm 14 \%)$. Após esse período, as juntas coladas foram seccionadas de acordo com a Norma
ASTM D 2339-93, para a obtenção dos corpos-de-prova, que foram condicionados à temperatura de $\pm 20^{\circ} \mathrm{C} \mathrm{e}$ umidade relativa de $65 \%$. Após o equilíbrio, a resistência ao cisalhamento e a percentagem da falha na madeira foram determinadas.

\subsection{Análise estatística dos dados}

Para verificar o efeito da hidrólise nas propriedades dos adesivos tânicos foi instalado um experimento, seguindo arranjo fatorial completo, com três tempos de reação e quatro valores de $\mathrm{pH}$, num total de 12 tratamentos, com oito repetições. Os resultados foram submetidos à análise de variância (ANOVA). Quando estabelecidas diferenças significativas, os tratamentos foram comparados entre si, por meio do teste de Tukey a 5\% de significância.

\section{RESULTADOS E DISCUSSÃO}

\subsection{Propriedades dos adesivos tânicos de Eucalyptus grandis hidrolisados com ácido acético}

\subsubsection{Teor de sólidos}

A análise de variância indicou que o teor de sólidos dos adesivos de taninos de Eucalyptus grandis foi afetado pelos tratamentos, ocorrendo interações significativas entre o tempo de reação e o $\mathrm{pH}$ de hidrólise, a 5\% de significância, pelo teste F.

Observa-se, pela Tabela 1, que os teores de sólidos para os adesivos produzidos com taninos reagidos durante 30 min foram iguais, com exceção do teor de sólidos do adesivo, produzido com taninos com pH igual a 3 . Para os adesivos produzidos com o tempo de reação de 60 min, verificou-se que aqueles com pH 5 e 6 obtiveram teor de sólidos significativamente iguais. O teor de sólidos dos adesivos produzidos com taninos hidrolisados em pH 3 e 4 foram significativamente diferentes entre si. Os teores de sólidos dos adesivos fabricados com o tempo de reação de 90 min diferiram significativamente nos diferentes valores de $\mathrm{pH}$ dos taninos, com exceção do adesivo fabricado com os taninos brutos, em que o teor de sólidos foi igual ao adesivo com pH 4.

Ainda na Tabela 1, estudando o efeito do tempo de reação para fabricação dos adesivos, dentro de cada valor de $\mathrm{pH}$ de hidrólise dos taninos, sobre o teor de sólidos, verifica-se que não houve diferenças significativas no teor de sólidos dos adesivos fabricados com taninos em pH 5 e 6, nos diferentes tempos de reação. Para aqueles hidrolisados em $\mathrm{pH}$ igual a 4, o tempo de reação

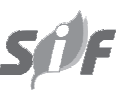

R. Árvore, Viçosa-MG, v.33, n.4, p.733-739, 2009 
de 30 min apresentou o maior teor de sólidos. Os teores de sólidos dos adesivos produzidos com taninos hidrolisados em $\mathrm{pH} 3$ foram significativamente diferentes nos três tempos de reação utilizados para produzir os adesivos, tendo o adesivo produzido no tempo de reação de 30 min apresentado o maior teor de sólidos.

De modo geral, os adesivos produzidos com taninos hidrolisados em pH 3 apresentaram os menores valores médios de teor de sólidos em razão, principalmente, da maior adição de ácido para alcançar o pH desejado.

\subsubsection{Tempo de gelatinização}

A análise de variância indicou que o tempo de gelatinização dos adesivos de taninos de Eucalyptus grandis foi afetado pelos tratamentos, ocorrendo interações significativas entre o tempo de reação e o pH de hidrólise, a 5\% de significância, pelo teste F.

Observa-se, na Tabela 2, que no tempo de reação de 30 min o tempo de gelatinização dos adesivos foi significativamente diferente, sendo maior o tempo de gelatinização do adesivo produzido com taninos hidrolisados com pH igual a 3. No tempo de reação de 60 e 90 min, nota-se, nessa tabela, que não houve diferenças significativas no tempo de gelatinização entre os adesivos produzidos com taninos com $\mathrm{pH}$ 5 e 6 (não hidrolisado). Os adesivos produzidos com taninos hidrolisados com pH 3 apresentaram polimerização mais lenta que a dos demais.

Com exceção dos adesivos à base de taninos com pH igual a 4 e 6 (não-hidrolisado), o tempo de gelatinização não diferiu significativamente nos diferentes tempos de reação dos taninos para a produção dos adesivos.

\subsubsection{Viscosidade}

A análise de variância indicou que a viscosidade dos adesivos de taninos de Eucalyptus grandis foi afetada pelos tratamentos, ocorrendo interações significativas entre o tempo de reação e o pH de hidrólise, a 5\% de significância, pelo teste F.

Analisando a Tabela 3, observa-se que nos adesivos produzidos com taninos hidrolisados com pH 3 a viscosidade diminuiu com o aumento do tempo de reação, tendo diferenças significativas entre eles. Nos adesivos produzidos com taninos hidrolisados com $\mathrm{pH} 4$, notase, nessa tabela, que não houve diferenças significativas na viscosidade entre os adesivos produzidos com taninos reagidos durante 30 e $60 \mathrm{~min}$. A viscosidade dos adesivos produzidos no tempo de reação de 60 min também não diferiu, estatisticamente, dos tempos de 30 e $90 \mathrm{~min}$. As menores viscosidades foram encontradas nos adesivos produzidos com taninos hidrolisados com $\mathrm{pH} 3$ e 6 , nos tempos de reação de 90 e $30 \mathrm{~min}$, respectivamente.

Tabela 1 - Valores médios de teor de sólidos (\%) dos adesivos produzidos com taninos de Eucalyptus grandis, em função do $\mathrm{pH}$ e do tempo de reação

Table 1 - Average values of solid content (\%) for the adhesives produced with Eucalyptus grandis tannins, according to the $\mathrm{pH}$ and the time of reaction

\begin{tabular}{ccccc}
\hline Tempo de reação (minutos) & \multicolumn{3}{c}{ Teor de sólidos (\%) } \\
\cline { 2 - 5 } & $\mathrm{pH} \mathrm{3}$ & $\mathrm{pH} \mathrm{4}$ & $\mathrm{pH} \mathrm{5}$ & pH 6 (não-hidrolisado) \\
\hline 30 & $41,33 \mathrm{Ba}$ & $45,39 \mathrm{Aa}$ & $45,70 \mathrm{Aa}$ & $44,85 \mathrm{Aa}$ \\
60 & $40,43 \mathrm{Cb}$ & $44,22 \mathrm{Bb}$ & $45,29 \mathrm{Aa}$ & $44,37 \mathrm{Aba}$ \\
90 & $38,33 \mathrm{Cc}$ & $43,60 \mathrm{Bb}$ & $45,54 \mathrm{Aa}$ & $44.37 \mathrm{Ba}$ \\
\hline
\end{tabular}

Médias ao longo das linhas seguidas das mesmas letras maiúsculas e ao longo das colunas seguidas das mesmas letras minúsculas não diferem significativamente entre si, pelo teste de Tukey a $5 \%$ de significância.

Tabela 2 - Valores médios de tempo de gelatinização (s) dos adesivos produzidos com taninos de Eucalyptus grandis, em função do $\mathrm{pH}$ e do tempo de reação

Table 2 - Average values of gel time (s) for the adhesives produced with Eucalyptus grandis tannins, according to the pH and time of reaction

\begin{tabular}{ccccc}
\hline Tempo de reação (minutos) & \multicolumn{4}{c}{ Tempo de gelatinização (s) } \\
\cline { 2 - 5 } & $\mathrm{pH}$ & $\mathrm{pH} \mathrm{4}$ & $\mathrm{pH} \mathrm{5}$ & $\mathrm{pH} \mathrm{6}$ (não-hidrolisado) \\
\hline 30 & $106,17 \mathrm{Aa}$ & $81,83 \mathrm{Bb}$ & $61,17 \mathrm{Da}$ & $72,00 \mathrm{Ca}$ \\
60 & $111,67 \mathrm{Aa}$ & $99,08 \mathrm{Ba}$ & $55,25 \mathrm{Ca}$ & $51,00 \mathrm{Cb}$ \\
90 & $110,42 \mathrm{Aa}$ & $93,50 \mathrm{Ba}$ & $64,92 \mathrm{Ca}$ & $56,33 \mathrm{Cb}$ \\
\hline
\end{tabular}

Médias ao longo das linhas seguidas de mesmas letras maiúsculas e ao longo das colunas seguidas de mesmas letras minúsculas não diferem significativamente entre si pelo teste Tukey, a $5 \%$ de significância.

R. Árvore, Viçosa-MG, v.33, n.4, p.733-739, 2009 
Tabela 3 - Valores médios de viscosidade (cP) dos adesivos produzidos com taninos de Eucalyptus grandis, em função do $\mathrm{pH}$ e do tempo de reação

Table 3 - Average values of viscosity ( $(P)$ for the adhesives produced with Eucalyptus grandis tannins, according to the pH and time of reaction

\begin{tabular}{ccccc}
\hline Tempo de reação (minutos) & \multicolumn{3}{c}{ Viscosidade (Cp) } \\
\cline { 2 - 5 } 30 & $\mathrm{pH} 3$ & $\mathrm{pH}$ & $\mathrm{pH} \mathrm{5}$ & pH 6 (não-hidrolisado) \\
60 & $3.068 \mathrm{Ca}$ & $5.309 \mathrm{Ba}$ & $8.112 \mathrm{Ab}$ & $1.968 \mathrm{Db}$ \\
90 & $2.087 \mathrm{Cb}$ & $4.734 \mathrm{Bab}$ & $9.375 \mathrm{Aa}$ & $10.000 \mathrm{Aa}$ \\
\hline
\end{tabular}

Médias ao longo das linhas seguidas de mesmas letras maiúsculas e ao longo das colunas seguidas de mesmas letras minúsculas não diferem significativamente entre si pelo teste Tukey, a 5\% de significância.

Observa-se, na Tabela 3 , que os adesivos produzidos com taninos com pH 6 (não hidrolisado) apresentaram os maiores valores de viscosidade, à exceção daquele com o tempo de reação de $30 \mathrm{~min}$. Também, nota-se que a viscosidade dos adesivos produzidos com taninos em $\mathrm{pH} 3,4$ e 5 foi diferente da dos demais.

\subsection{Resistência ao cisalhamento na linha de cola}

A análise de variância indicou que a resistência ao cisalhamento foi afetada pelos tratamentos, ocorrendo interações significativas entre o tempo de reação e o pH de hidrólise dos taninos.

Pode-se constatar, pela Tabela 4, que a resistência ao cisalhamento na linha de cola dos adesivos produzidos com taninos reagidos durante 30 min não foi afetada pelos diferentes valores de $\mathrm{pH}$ de hidrólise dos taninos, exceto aqueles produzidos com $\mathrm{pH}$ igual a 5. Nos adesivos produzidos com tempo de reação de $60 \mathrm{~min}$, verificase que aqueles produzidos em $\mathrm{pH}$ igual a 6 (não hidrolisado) apresentaram maior resistência, diferindo significativamente dos demais. Verificou-se também que, no tempo de $90 \mathrm{~min}$, a resistência ao cisalhamento aumentou com maiores valores de $\mathrm{pH}$ de hidrólise, exceto com pH igual a 6.
Verificou-se ainda que, de modo geral, a resistência ao cisalhamento dos adesivos produzidos com taninos em pH 3 e 4 e reagidos durante $30 \mathrm{~min}$ foi maior que a resistência dos adesivos produzidos nos demais tempos de reação dos taninos. Não foram verificadas diferenças significativas na resistência dos adesivos produzidos com taninos hidrolisados com $\mathrm{pH} 5$ e tempo de reação de 30 e $90 \mathrm{~min}$. No geral, de acordo com os dados desta pesquisa, o tempo de reação utilizado para produção dos adesivos não teve influência sobre a resistência ao cisalhamento.

Mori et al. (1999), avaliando a resistência dos adesivos de taninos de Eucalyptus grandis, encontraram resistência média igual a 1,5 MPa. De modo geral, nos adesivos produzidos nesse experimento com taninos hidrolisados de Eucalyptus grandis esses valores de resistência foram aumentados, em média, para 3,6 MPa.

\subsubsection{Falha na madeira}

A análise de variância indicou que a falha da madeira na linha de cola dos adesivos de taninos de Eucalyptus grandis foi afetada pelos tratamentos, ocorrendo interações significativas entre o tempo de reação e pH de hidrólise dos taninos, a 5\% de significância, pelo teste F.

Tabela 4 - Valores médios de resistência ao cisalhamento (MPa) para os adesivos produzidos com taninos de Eucalyptus grandis, em função do $\mathrm{pH}$ e do tempo de reação

Table 4 -Average values of shear resistance (MPa) for the adhesives produced with Eucalyptus grandis tannins, according to the $\mathrm{pH}$ and time of reaction

\begin{tabular}{ccccc}
\hline Tempo de reação (minutos) & \multicolumn{4}{c}{ Resistência ao cisalhamento (MPa) } \\
\cline { 2 - 5 } & $\mathrm{pH} \mathrm{3}$ & $\mathrm{pH} \mathrm{4}$ & $\mathrm{pH} \mathrm{5}$ & $\mathrm{pH} \mathrm{6}$ (não-hidrolisado) \\
\hline 30 & $3,732 \mathrm{Aa}$ & $3,817 \mathrm{Aa}$ & $3,651 \mathrm{Ba}$ & $3,879 \mathrm{Aa}$ \\
60 & $3,368 \mathrm{Bb}$ & $3,358 \mathrm{Bb}$ & $3,452 \mathrm{Bb}$ & $3,696 \mathrm{Aa}$ \\
90 & $3,276 \mathrm{Cb}$ & $3,528 \mathrm{Bb}$ & $3,808 \mathrm{Aa}$ & $3,155 \mathrm{Cb}$ \\
\hline
\end{tabular}

Médias ao longo das linhas seguidas de mesmas letras maiúsculas e ao longo das colunas seguidas de mesmas letras minúsculas não diferem significativamente entre si pelo teste Tukey, a $5 \%$ de significância. 
Observa-se, na Tabela 5, que a porcentagem de falha na madeira do adesivo produzido com taninos reagidos durante 30 min e pH igual a 6 (não hidrolisado) foi significativamente diferente, apresentando a maior porcentagem de falha. Nos adesivos produzidos com os taninos reagidos durante $60 \mathrm{~min}$, verificou-se que a maior porcentagem de falha na madeira ocorreu no adesivo produzido com taninos hidrolisados com $\mathrm{pH}$ igual a 3. Não houve diferenças significativas na porcentagem de falha na madeira quanto aos adesivos produzidos com o tempo de reação de $90 \mathrm{~min}$.

Os adesivos tânicos produzidos com o tempo de reação de 30 min apresentaram as maiores porcentagens de falha na madeira. Esses valores foram maiores que aqueles encontrados por Mori et al. (2001), que constataram que a falha na madeira com relação aos adesivos de taninos de Eucalyptus grandis ficou abaixo dos $10 \%$.

Tabela 5 - Valores médios de falha na madeira para os adesivos produzidos com taninos de Eucalyptus grandis, em função do $\mathrm{pH}$ e do tempo de reação

Table 5 - Average values of wood failure for adhesives produced with Eucalyptus grandis tannins, according to the pH function and the time of reaction

\begin{tabular}{ccccc}
\hline Tempo de reação (minutos) & \multicolumn{4}{c}{ Falha na madeira (\%) } \\
\cline { 2 - 5 } & $\mathrm{pH} \mathrm{3}$ & $\mathrm{pH}$ & $\mathrm{pH} \mathrm{5}$ & $\mathrm{pH} \mathrm{6}$ (não-hidrolisado) \\
\hline 30 & $17,74 \mathrm{Ba}$ & $18,32 \mathrm{Ba}$ & $15,70 \mathrm{Ba}$ & $31,68 \mathrm{Aa}$ \\
60 & $21,33 \mathrm{Aa}$ & $10,30 \mathrm{Bb}$ & $2,68 \mathrm{Cb}$ & $2,52 \mathrm{Cb}$ \\
90 & $1,84 \mathrm{Ab}$ & $3,13 \mathrm{Ac}$ & $4,48 \mathrm{Ab}$ & $4,22 \mathrm{Ab}$ \\
\hline
\end{tabular}

Médias ao longo das linhas seguidas de mesmas letras maiúsculas e ao longo das colunas seguidas de mesmas letras minúsculas não diferem significativamente entre si pelo teste Tukey, a $5 \%$ de significância.

\section{CONCLUSÕES}

O pH de hidrólise dos taninos afetou as propriedades dos adesivos, e aqueles produzidos com taninos hidrolisados com pH igual a 3 apresentaram menor viscosidade e maior tempo de gelatinização, devido à quebra das ligações interflavonoides e à hidrólise de substâncias não tânicas.

De modo geral, a redução do $\mathrm{pH}$ dos taninos não colaborou para o aumento da resistência ao cisalhamento na linha de cola, no entanto melhorou as suas propriedades de trabalhabilidade.

O aumento no tempo de reação dos taninos reduziu o teor de sólidos e a viscosidade dos adesivos produzidos em pH 3 e 4 . O tempo de gelatinização dos adesivos não foi afetado pelo tempo de reação, exceto nos produzidos com o tempo de $30 \mathrm{~min}$ nos $\mathrm{pH} 4$ e 6 . Assim, a melhor adesão foi verificada nos adesivos produzidos com taninos hidrolisados com pH igual a 3 e tempo de reação de $30 \mathrm{~min}$, apresentando melhor aplicabilidade e maior resistência ao cisalhamento.

As juntas coladas com os adesivos tânicos de Eucalyptus grandis apresentaram baixo percentual de falha na madeira.

Conclui-se, de modo geral, que tempos longos de reação aliados a baixos valores de $\mathrm{pH}$ dos taninos degradam demasiadamente as suas moléculas, não contribuindo, de forma satisfatória, para o aumento da resistência ao cisalhamento e, consequentemente, para o aumento no percentual de falha na madeira.

Recomendam-se outros estudos com diferentes tipos de ácidos e concentrações, de modo a determinar o ponto de equilíbrio entre a viscosidade, o teor de sólidos e a resistência, mantendo o tempo de trabalho dos adesivos suficiente para aplicação e economicamente viável quanto à polimerização. Recomenda-se, também, um estudo desses adesivos hidrolisados através da análise de cromatografia de permeação em gel e calorimetria diferencial exploratória para determinação do peso molecular e da temperatura de cura, respectivamente.

\section{REFERÊNCIAS}

AMERICANSOCIETYFOR TESTINGAND MATERIALS - ASTM. Annual Book of ASTM Standards, ASTM D -2339, v.4.10. Philadelphia: 1993.

BISANDA. E. T. N.; OGOLAW, O.; TESHA, J. V. Characterization of tanin resin blends for particle board applications. Cement \& Concrete Composites, v.25, n.6, p.593-598, 2003.

CARNEIRO, A. C. O. et al. Propriedades de chapas de flocos fabricadas com adesivo de uréiaformaldeído e de taninos de Eucalyptus grandis W. Hill ex Maiden e de Eucalyptus pellita F. Muell. Revista Árvore, v.28, n.5, p.715-724, 2004. 
CARNEIRO, A. C. O. Efeito da sulfitação dos taninos de $E$. grandis $e$ E. pellita para produção de chapas de flocos. 2002. $91 \mathrm{f}$. Dissertação (Mestrado em Ciência Florestal) Universidade Federal de Viçosa, Viçosa, MG, 2002.

MORI, C. et al. Uso de taninos da casca de três espécies de Eucalyptus na produção de adesivos para colagem de madeira. Revista Árvore, v.25, n.1, p.19-28, 2001.

MORI, F. A. Caracterização parcial dos taninos da casca e dos adesivos de três espécies de eucaliptos. 2000. 73f. Tese (Doutorado em Ciência Florestal) - Universidade Federal de Viçosa, Viçosa, MG, 2000.

MORI, F. A. et al. Utilização de resinas a base de taninos da casca de Eucalyptus grandis W. Hill ex Maiden na produção de painéis compensado. Revista Árvore, v.23, n.4, p.455-462, 1999.
Moslemi, A. A. Particleboard.

Carbondale: Southern Illinois University Press, 1974. v.1.243p.

SIMÃO, J. B. P. Efeito de diferentes inóculos na compostagem de cascas de eucalipto em sistema de medas. 1992. 48f. Dissertação (Mestrado emCiência Florestal) - Universidade Federal de Viçosa, Viçosa, 1992.

SOWUNMI, S. et al. Fortified mangrove tanninbased adhesive. Journal of Applied Polymer Science, v.62, n.3, p.577-584, 1996.

SOWUNMI, S. et al. Differential scanning calorimetry of hydrolysed mangrove tannin. Polymer International, v.49, n.6, p.574-578, 2000. 